\title{
Phase and microphase separation in polymer blends
}

\author{
M. DAOUD
}

Laboratoire Léon Brillouin, CEA/CNRS, CEN Saclay, 91191 Gif-sur-Yvette cedex, France

\begin{abstract}
When two different polymers are mixed, they usually demix because of the slight repulsive interaction that exists between their monomers. This is general and may be related to phase separation in two component mixtures. When the same polymer blend is cross-linked in the one phase region, elastic forces develop and do not let this to occur. There results a microphase separation, with coexistence of regions of finite extent and different compositions. We discuss these phenomena as well as the kinetics of the decomposition.
\end{abstract}

\section{Introduction.}

For many practical purposes, blends of two -or more- polymers are interesting because they combine properties that are not present for a single macromolecular species. However, most usually, there is a small interaction between the monomers leading to a phase separation of the mixture(1)-(3). This phase separation occurs even when the monomer-monomer interaction is very small, and is related to the fact that each polymer contains a large number of units. Thus even though the monomer-monomer interaction is small, the interchain interaction may be large, leading to a phase separation. The coexistence diagrams of the different species were determined both theoretically and experimentally, with a good agreement. The kinetics of these phase separations was also carefully studied more recently(4)-(7). Because of the large masses of the polymers, the times are usually much larger then in the case of simple liquid mixtures. This allowed for a careful study of the various stages of the decomposition. Some of these are still not completely understood.

Because of the usefulness of polymer mixtures however, other ways were looked for in order to get a larger coexistence region for polymers with different properties. One original way that was found is to get the chains cross-linked in order to get a gel made of both species, or two interpenetrated networks(IPNs), each being made of one of the component polymers. This is typically what is nowadays called an interpenetrating network(8)(9). Many different ways were used chemically to get these networks, corresponding to a complicated 
nomenclature. The reason for such carefulness in the description of these networks is related to the uncertainty concerning the fact that the products may not be in thermodynamic equilibrium. A typical way to get an IPN is to make a first gel. This is swollen by the monomer of the second gel. Finally gelation of the second monomer is performed in order to get the IPN. This is obviously an idealized description. It neglects completely the eventual existence of a phase separation as the last step proceeds. The actual result is rather the result of a competition between the kinetics of the second gelation process that we just mentioned, and the kinetics of the phase separation that occurs when the final step proceeds. Recently however, de Gennes(10) gave a first tentative model that seems to capture the fundamentals of the underlying physics in these IPNs. In this model, one starts with a mixture of two linear polymers in the one phase region. These chains are cross-linked in order to get a tight gel, with distance d between cross-links much smaller than the distance a between entanglements. Finally, the system is brought to a lower temperature, in the two phase region. Because of the cross-links, there develops an elastic restoring force that counteracts the tendency of the system to phase separation. As a result, there is a microphase separation that takes place instead of the usual macroscopic phase separation. This implies a better mixing of the component chains, because the demixing occurs only at finite distances.

In what follows, we will first describe the thermodynamic properties of the phase separation that happens in polymer mixtures. Then we will turn to de Gennes' model for the microphase separation that is present in cross-linked blends. We will also discuss the influence of cross-linking density on the microphase separation. Finally, we will consider the kinetics of both phenomena.

2. Polymer blends.

Let us consider a polymer mixture made of two different species $A$ and $B$. We will assume for simplicity that both are made of the same number $N$ of units. This assumption will simplify the calculations without altering the main results significantly. Let $\Phi$ be the fraction of A monomers, 1- $\Phi$ the fraction of B units, and $\chi$ the interaction between $A$ and $B$ monomers. The simplest way to write the free energy $F$ per unit volume of the mixture was given some years ago by Flory and Huggins $(1,2)$ and may be given in the following way

$$
F=\frac{\Phi}{N} \operatorname{Ln} \Phi+\frac{1-\Phi}{N} \operatorname{Ln}(1-\Phi)+\chi \Phi(1-\Phi)
$$

where the energies are measured in units $k_{B} T$. The first two terms are the entropy contributions of the center of masses of the polymers considered as ideal gases. The last term is the interaction energy. The field conjugate to the fraction $\Phi$ , that will be called chemical potential below, $\mu$ is

$$
\mu=\frac{\partial \mathrm{F}}{\partial \Phi}=\frac{1}{\mathrm{~N}} \operatorname{Ln} \frac{\Phi}{1-\Phi}+\chi(1-2 \Phi)
$$

The coexistence curve is obtained by equating the chemical potential to zero, spinodal curve by equating to zero the derivative of $\mu$ with respect to $\Phi$. 


$$
\frac{1}{\mathrm{~N} \Phi(1-\Phi)}-2 \chi=0
$$

The critical point is obtained by equating to zero its second derivative:

$$
\frac{1}{N}\left\{\frac{1}{(1-\Phi)^{2}}-\frac{1}{\Phi^{2}}\right\}=0
$$

Equations (3) and (4) together give the location of the critical point. We find

$$
\begin{aligned}
& \Phi_{c}=1 / 2 \\
& \chi_{c}=2 / \mathrm{N}
\end{aligned}
$$

Let us mention here that the interaction parameter $\chi$ is directly related to the temperature $T$ of the system. It is usually assumed that $\chi$ is inversely proportional to $T$. This is because the monomer-monomer interaction is assumed to be independent of $T . \chi$ is such energy measured in units of $k_{B} T$ :

$$
\chi=\frac{\chi_{0}}{T}
$$

It is then possible to expand the free energy, relation (1), in the vicinity of the critical point. Let $\phi$ be the fraction difference between the actual fraction $\Phi$ and the critical one: $\phi=\Phi-1 / 2$. We find

$$
F=\left(\chi_{c}-\chi\right) \phi^{2}+0\left(\phi^{4}\right)
$$

So far, the free energy did not take into account the spatial variations of the volume fraction related to the presence of interfaces between $A$ rich and $B$ rich phases. In order to include this, one includes in relation (1) a gradient term corresponding to these variations. Introducing a position dependent concentration $\Phi(\mathbf{r})$, this gives instead of (1)

$$
F=\int\left\{\left(\chi_{c}-\chi\right) \phi(\mathbf{r})^{2}+a^{2}(\nabla \phi(\mathbf{r}))^{2}\right\} d \mathbf{r}
$$

Introducing the Fourier transform of $\phi(\mathbf{r})$

$$
\phi(q)=\int_{\phi(r)} e^{i q \cdot r} d r
$$

relation (8) may be written in reciprocal space as 


$$
F=\sum_{q}\left\{\left(\chi_{c}-\chi\right)+q^{2} a^{2}\right\}\left|\rho_{q}\right|^{2}
$$

Introducing the reduced temperature

$$
\varepsilon=\frac{\chi_{\mathrm{c}}-\chi}{\chi_{\mathrm{c}}}
$$

relation (10) becomes

$$
F=\sum_{q} \frac{1}{N}\left\{\varepsilon+q^{2} R^{2}\right\}\left|\rho_{q}\right|^{2}
$$

where

$$
\mathrm{R}^{2} \sim \mathrm{Na}^{2}
$$

is the radius of gyration of the polymers. Relation (11) is the familiar Landau expression for the free energy in a second order transition. Thus the above approach is equivalent to the usual mean- field theory for the critical point. Note that the characteristic length is proportional to the radius $R$ of the chains rather than to the monomer size a.

Relation (11) allows us to determine all the properties of the coexistence curve near the critical point exactly as in the conventional Landau theory. Thus we find that the coexistence curve is

$$
\rho \sim \varepsilon^{1 / 2}
$$

and the correlation function

$$
\mathrm{S}(\mathrm{q}) \sim\left\langle|\rho(\mathrm{q})|^{2}\right\rangle \sim \frac{1}{\varepsilon+\mathrm{q}^{2} \mathrm{R}^{2}}
$$

Therefore the correlation length $\xi$ in the problem is

$$
\xi \sim R \varepsilon^{-1 / 2}
$$

and diverges at the critical point. Note the presence of a large prefactor $R$ that makes the correlation length much larger than for the case of a simple binary fluid. Relation (14) was checked with several polymer mixtures. That mean field is valid for a polymer blend is not a surprise because of the presence of very large objects that are overlaping in order to fill space. Using relation (12), it is straightforward to show that in a sphere with radius $R$, there are $N^{1 / 2}$ chains. Therefore a given chain has on the average this number of other chains of the other species in its immediate neighborhood, and interacts with them. In other words, evry chain has a large number of neighbors with which it is interacting. It 
was shown for magnetic systems that in such case, mean field theory is valid. This may be made more precise by calculating the fluctuations, i.e. $\left\langle\phi^{2}>/\left(\phi-\phi_{c}\right)^{2}\right.$. The denominator is $\left(\chi_{c}-\chi\right)$. The numerator is evaluated from relation (14) for $q R$ $\rightarrow 0$ and is of the order of $\left(\chi_{c}-\chi\right)^{-1}$. Therefore the fluctuations areof the order of $\left(\chi_{c}-\chi\right)^{-2} \sim(N / \varepsilon)^{2}$, with $\varepsilon=\left(\chi_{c}-\chi\right) / \chi_{c}$. Thus as long as $\varepsilon>\varepsilon^{*} \sim N^{-1}$, the fluctuations are small and mean field is valid.

For an asymmetric mixture, when the lengths $N_{A}$ and $N_{B}$ of the species are different, the phase diagram become non symmetric and the width of the critical region increases. We will merely mention the result for the limiting case when one of the species is a simple solvent. Then critical point is, instead of relations (5) above

$$
\begin{aligned}
& \Phi_{c} \sim \mathrm{N}^{-1 / 2} \\
& \theta-\mathrm{T}_{\mathrm{c}} \sim \mathrm{N}^{-1 / 2}
\end{aligned}
$$

where $\theta$ is the Flory compensation temperature where the chain has gaussain behavior.

\section{Microphase separation.}

As mentioned in the introduction, it is interesting to consider the case when phase separation is inhibited by cross-linking of the various polymers and the formation of interpenetrating networks. An interesting model was introduced recently by de Gennes in order to consider one possible equilibrium state of such mixtures. In this model, one considers the mixture of A and B chains in the one phase region. The various polymers are cross-linked in such a way that one gets a tight gel. The mesh size is assumed to be smaller than the distance between entanglement points. Finally, the temperature of the system is lowered, so that one gets in the vicinity of the critical point that was considered in the previous section, where the uncross-linked system would have phase separated. In order to describe such system, de Gennes(10) added to the previous free energy an elastic contribution.

$$
F=\sum_{q}\left\{\left(\chi_{c}-\chi\right)+q^{2} a^{2}\right\}\left|\rho_{q}\right|^{2}+C P^{2}
$$

where $C$ is an elastic constant to be discussed below. The origin of the last term in the free energy lies in the fact that the system is cross-linked now. Therefore when phase separation is initiated by lowering the temperature, the various chains that had to migrate in order to phase separate are unable to do so because they are cross-linked. In order to calculate this elastic contribution, de Gennes developed an analogy with dielectric media. If we assume that each of the polymers is electrically charged, with opposite charges for each of the chemical species in order to preserve total neutrality of the system, when phase separation proceeds, we get charge fluctuations, and regios that are charged either positively or negatively. In a dielectric medium, we know that a 
polarization $\mathbf{P}$ appears. The latter may be related in the problem that we are discussing to the average distance between the centers of masses of the various polymers. In the dielectric medium, the polarization is related to the charge density

$$
\operatorname{div} \mathbf{P}=-\rho
$$

In the equivalence, this charge density may be directly related to the density fluctuation $\phi$. Therefore, we write formally

$$
\operatorname{div} \mathbf{P}=-\phi
$$

Fourier transforming the latter relation, and inserting in equation (17), we get

$$
F=\sum_{q}\left\{\left(x_{c}-x\right)+q^{2} a^{2}+\frac{C}{q^{2}}\right\}\left|\rho_{q}\right|^{2}
$$

Note that because of relation (18), only longitudinal modes are considered now. The latter are modes where $q$ is parallel to $P_{q}$. It is then possible to determine the correlation function, or its Fourier transform

$$
S(q) \sim\left\{\left(x_{c}-x\right)+q^{2} a^{2}+\frac{C}{q^{2}}\right\}^{-1}
$$

This function exhibits a maximum for a value $q^{*}$ of the wavevector related to the elastic constant

$$
\mathrm{q}^{*} \sim \mathrm{C}^{-1 / 4}
$$

and

$$
\mathrm{S}\left(\mathrm{q}^{*}\right)-\left\{\left(\chi_{\mathrm{c}-}-\chi\right)+\mathrm{q}^{*} \mathrm{a}^{2}\right\}^{-1}
$$

This maximum diverges at the value $\chi_{\text {s }}$ corresponding to the spinodal temperature

$$
\chi_{\mathrm{s}}-\chi_{\mathrm{c}} \sim \mathrm{q}^{*^{2}} \mathrm{a}^{2}
$$

Thus two conclusions may be drawn: as long as $q^{*}$ is finite, a microphase separation occurs. The latter involves finite regions in space rather than macroscopic demixion, as was the case in the absence of cross-linking.

There is also a temperature shift in the critical temperature, which goes from $\chi_{c}$ in the uncross-linked case to $\chi_{s}$ in the cross-linked case. This shift also depends on the elastic constant $C$ which we evaluate now. This constant corresponds to the rigidity of the chains. For one chain, we know that it is 


$$
C_{1} \sim \frac{1}{R_{0}^{2}} \sim \frac{1}{N}
$$

For a part of a chain made of $\mathrm{n}$ monomers between two consecutive cross-links, we have a similar relation involving $n$. However, the free energy is evaluated per site. Because there are $\mathrm{n}$ monomers per strand, we get

$$
C \sim \frac{1}{n^{2}}
$$

Combining relations (21), (23) and (24b), we finally get

and

$$
\mathrm{q}^{*} \sim \mathrm{n}^{-1 / 2}
$$

$$
\chi_{\mathrm{s}}-\chi_{\mathrm{c}} \sim \mathrm{n}^{-1}
$$

Thus we find that $\chi_{s}$ is larger than $\chi_{c}$. This implies that the critical temperature for the microphase separation is lower than that for the macroscopic phase separation. Therefore the one phase region is larger for the cross-linked case than it was for the polymer mixture. Note also that the size of the microdomains is directly related to the distance between consecutive cross-links

The previous results were checked experimentally by Briber and Bauer(11) with a mixture of Polyvinyl-methyl-ether, mixed with deuterated polystyrene. The cross-linking was performed by irradiating the mixture. The main assumption in their experiment is that the chemical distance between crosslinks is inversely proportional to the irradiation dose. The experimental results were in reasonable agreement with the predictions, with the exception of the scattered intensity at zero angle that does not vanish as found above. Such discrepancy is related to the fact that the initial mixture is not random as assumed above, but has fluctuations that are frozen in by the irradiation. Work is in progress on this last point. Another extension concerns the influence of the density of cross-links on the microphase separation(12). As the number $\mathrm{n}$ of monomers is increased, the system eventually goes through a sol gel transition. Below this transition, one may ask whether the microphase separation still occurs or not. One realizes that there is no need for an infinite gel to be present in order to get the microphase separation. Indeed, the origin of the restoring elastic energy lies in the fact that two different polymers are linked to each other. As temperature decreases, these chains tend to phase separate. Therefore their center of masses tend to be no longer at the same location. This implies that the restoring force will be present even below the gelation threshold. A direct way to realize this might be to consider diblock copolymers, which are known to microphase separate at lower temperatures. Thus even when the probability $p$ that two chains are cross-linked is very low, the microphase separation occurs. For $p \rightarrow 0$, one goes back to the macroscopic phase separation. Let us note however that in this analysis, we neglect the fact that in the vicinity of the threshold, and below it there is an important fraction of the polymers that did not cross-link, and therefore tend to 
phase separate macroscopically. One possibility then for the reacted polymers is to localize at the interface between $A$ and $B$ rich phases. Such possibility has not been considered so far to our knowledge.Neglecting the latter, it is straightforward to calculate the size of the microdomains for any value of $p$ below the threshold: in these conditions, chains are attached to each other. The value for the elastic constant for a chain is

$$
\mathrm{C}_{1} \sim \mathrm{N}^{-1}
$$

In the sphere that contains one polymer, for density reasons there are $\mathrm{N}^{1 / 2}$ other chains. A fraction $p$ of these is reacted. Therefore the elastic constant per site is

$$
\mathrm{C} \sim \mathrm{N}^{-1}\left(\mathrm{pN}^{1 / 2}\right)\left(\mathrm{N}^{-3 / 2}\right) \sim \frac{\mathrm{p}}{\mathrm{N}^{2}}
$$

Relations (21) and (23) together with (27) give the temperature shift and the size of the microphases.

4. Kinetics.

The kinetics of the phase separation was considered by de Gennes(1), Pincus(2) and by Binder(3). They showed that that reptation(13) has a significant contribution to the kinetics. In other words, it was shown that for instance the scattered intensity in a radiation scattering experiment has two components, namely a fast, small amplitude contribution, and a slow, large amplitude one. The former corresponds to the local motion whereas the latter is related to the large scale creep of the chains. One defines a current $\mathbf{j}$ for species $A$. It is then possible to write down a continuity equation

$$
\frac{\partial \Phi}{\partial t}+\operatorname{div} \mathbf{j}=0
$$

where $t$ is time, and the current $\mathbf{j}$ is related to the chemical potential $\mu(r)=\partial F / \partial \phi$

$$
\mathbf{j}(\mathbf{r})=\int \Lambda\left(\mathbf{r}-\mathbf{r}^{\prime}\right) \nabla \mu(\mathbf{r}) \mathrm{d} \mathbf{r}
$$

where $\Lambda(r)$ is an Onsager coefficient to be discussed below. Fourier transforming the latter equation we get

$$
j(q)=\Lambda(q)(\nabla \mu)_{q}
$$

Finally, assuming an exponential decay for the fluctuation

$$
\delta \Phi(\mathrm{q}, \mathrm{t})=\delta \Phi(\mathrm{q}, 0) \mathrm{e}^{-\mathrm{t} / \tau_{\mathrm{q}}}
$$

\subsection{Phase separation.}


using relations (10), (28) and (29) and keeping only linear terms in the composition fluctuations $(14,15)$, we get

$$
\tau^{-1}(\mathrm{q})=-\frac{1}{\delta \Phi_{\mathrm{q}}} \frac{\partial\left(\delta \Phi_{\mathrm{q}}\right)}{\partial \mathrm{t}}=\mathrm{q}^{2} \Lambda(\mathrm{q})\left[\chi_{\mathrm{c}}-\chi+\mathrm{q}^{2}\right]
$$

When $\chi$ becomes larger than $\chi_{\mathrm{c}}$, the fluctuations with wavevector larger than some value have positive relaxation time, and thus decay with time. Those with small enough wavevector on the contrary have negative relaxation times. Therefore they increase with time. This implies that such wavelengths are unstable against any fluctuation. The smallest wavevector separating these regimes is

$$
\mathrm{q}_{\mathrm{i}} \sim\left(\chi-\chi_{\mathrm{c}}\right)^{1 / 2}
$$

In order to determine completely the growth of the unstable modes, we need to know the Onsager coefficient that we introduced above. When $q$ goes to zero, we find from relation (31) that

$$
\tau^{-1}(q)=q^{2} \Lambda(q)\left[\chi_{c}-\chi\right]
$$

Therefore the last two coefficients in the latter relation may be identified with a diffusion coefficient. The latter is evaluated far from the transition, for high temperatures. Then one may identify $\chi_{c} \Lambda(0)$ with the diffusion coefficient of the center of mass $(16,17)$. This leads to

$$
\Lambda(\mathrm{q}=0) \sim \mathrm{N}^{-1}
$$

For $\mathrm{qR}>1$, Pincus(2) argued that

$$
\Lambda(q) \sim(q N)^{-2}
$$

leading to

$$
\tau^{-1}(q)=N^{-2}\left[\chi_{c}-\chi\right] \sim N^{-3} t
$$

Therefore the motion is independent of $q$.

4.2. microphase separation.

Using the free energy relation (20) instead of (10) for the microphase separation case, we get

$$
\tau^{-1}(q) \sim q^{2} \Lambda(q)\left(\chi_{c}-\chi+q^{2}+C / q^{2}\right)
$$

Although relation (37) and (33) seem to be very similar, the time scales that are involved in both of them are very different. The macroscopic phase separation takes place for times on the order of the reptation time. The microphase 
separation occurs at much smaller distances. If we assume for the time being that the gel is tightly cross-linked, the time scales for the microphase separation are rather Rouse times, and are much shorter than the ones that we considered above. Therefore, the Onsager coefficient is rather a Rouse(13) diffusion coefficient in the present case than a diffusion coefficient in the tube, as it was in the preceding subsection. If $\mathrm{n}$ is the chemical distance along the polymer between successive cross-links, one gets

$$
\Lambda(q=0)-n^{-1}
$$

For larger values of $\mathrm{q}$, one probes smaller distances, so that one is led to consider parts of the chain made of $n_{q} \sim q^{-2}$ units. Generalizing relation (38), we get

$$
\Lambda\left(\mathrm{qn}^{1 / 2}>1\right) \sim \mathrm{q}^{2}
$$

This leads to the characteristic time

$$
\tau^{-1}(q)=q^{4}\left[x_{c}-\chi+q^{2}+\frac{C}{q^{2}}\right]
$$

Figure (1) shows the various cases that may be obtained for the characteristic frequency $\Omega_{1}(q)=\tau^{-1}(q) / q^{4}$. Figure (1a) shows the case for $C=0$, when no crosslinks are present, and for very early stages. For $\chi<\chi_{c}\left(\right.$ or $\left.T>T_{c}\right)$ all frequencies are positive and no instabilities are present. In the opposite case, $T<T_{\mathfrak{c}}$, we find that all modes with

$$
\mathrm{q}>\mathrm{q}_{\mathrm{c}} \sim\left(\chi-\chi_{\mathrm{c}}\right)^{1 / 2}
$$

are stable whereas the large wavelength modes with $\mathrm{q}<\mathrm{q}_{\mathrm{c}}$ are unstable: Any fluctuation with such wavelength grows with time.

For a cross-linked mixture, $\mathrm{C} \neq 0$, shown in figure (1b), the situation is somewhat different. For negative $t$, the curves exhibit a minimum which may be positive or negative. For $\chi<\chi_{s}$ all frequencies are positive, and no instability appears. We remind that

$$
\chi_{c}-\chi_{s} \sim-C^{1 / 2}
$$

For $\chi=\chi_{\mathrm{s}}$ the minimum vanishes, and this corresponds to the microphase separation. For lower temperatures, $\Omega_{1}(q)$ has a negative part corresponding to the presence of instabilities in a finite $q$ range.

For large chains and a less tightly bound mixture, when the contour length $n$ between successive cross-links is larger than the length $\mathrm{N}_{\mathrm{e}}$ between entanglements, the kinetics involves local reptation in tube portions between cross-links. Therefore the early stages we are discussing are going to take place 
221

at longer times than the ones that were discussed above. The Onsager coefficient $\Lambda(q)$ corresponds no longer to a Rouse dynamics, but to the local reptation of the strands inside the tube portions. It was shown recently by Pincus (2) that such motion implies



Figure la: The relaxation rate as a function of wave number. For an uncross-linked mixture. For $\mathrm{t}<0$, low q modes are unstable.

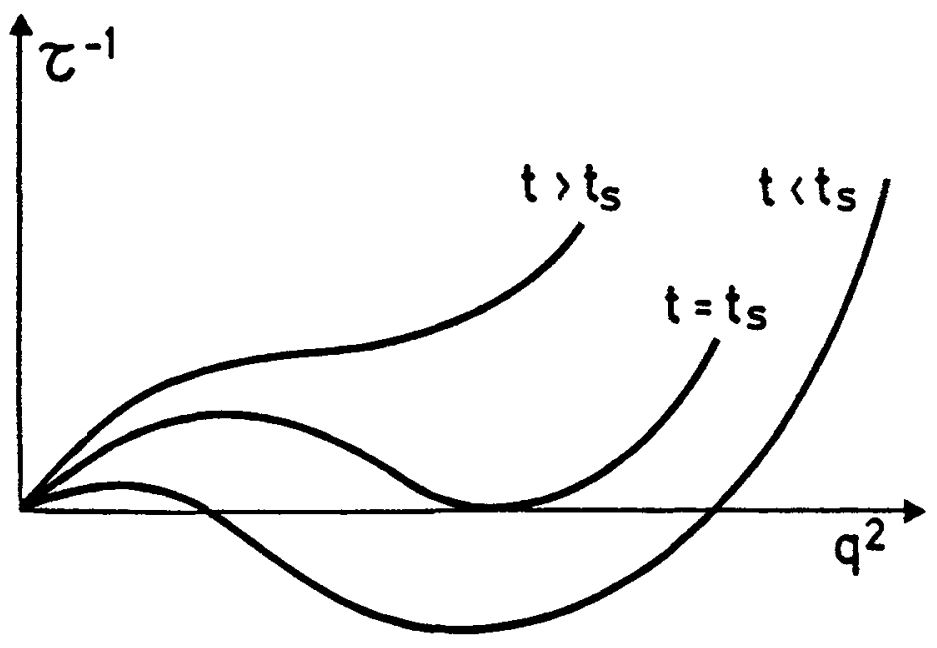

Figure ib: The relaxation rates for a cross-linked blend. For $0<t<t s$ no instabilities are present. Below ts modes are unstable in a range of $q$.

$$
\Lambda(q) \sim(q n)^{-2}
$$

and therefore

$$
\tau^{-1}(q)-n^{-2}\left(x_{c}-x+q^{2}+C / q^{2}\right)
$$


the cross-over between relations (44) and(36) occurring for $\mathrm{n} \sim \mathrm{N}_{\mathrm{e}}$. Relation (27) implies that the fastest growing instability is for $\quad \mathrm{q} \sim \mathrm{q}^{*}$, and grows as

$$
\tau^{-1}(q) \sim n^{-2}\left(x_{s}-\chi\right)
$$

Note that when $\mathrm{C}$ goes to zero, the latter relation crosses over smoothly to the Pincus result for a demixing (uncross-linked) polymer mixture, relation (36).

\section{Conclusion.}

We discussed the phase separation that occurs in polymer blends as well as its kinetics in the early stages, when linearization is valid. For the static properties, an important point is the fact that the radius of gyration of the polymers acts as a unit length in the problem. This leads to the fact that mean field is valid. Similarly, for the kinetics, because the reptation time is long, it was possible to actually observe the kinetics in the early stages, whereas this is much more difficult for simple liquid mixtures.

By cross-linking the polymers, phase separation occurs only locally, and this corresponds to microphase separation. This implies that the scattered intensity in a neutron scattering experiment has a maximum and that the intensity at the maximum diverges at the spinodal. The latter behavior is to be contrasted with the singularity for $\mathrm{q}=0$ in the case of macroscopic phase separation. Moreover, the spinodal temperature is lowered significantly in a cross-linked system when compared with the mixture. The distances involved in the microphase separation are ruled by the density of cross-links and may be much smaller than the radius of gyration of the polymers. Similarly, the kinetics is for much smaller times than for phase separation. When the density of cross-links is high, local Rouse dynamics is valid. As the number of cross-links decreases, local reptation as discussed by Pincus becomes valid, and the kinetics becomes similar to phase separation at very early times.

Finally, we note that this is a simple equilibrium model, that may certainly not describe the diversity of the various ways of synthesizing interpenetrated networks. One may also question the validity of any equilibrium model for such description: some IPNs may not be at equilibrium. This might be checked by annealing experiments for instance.

\section{Acknowledgments.}

The author has benefited from discussions with M. Benhamou, A. Brulet, H. Frisch, and L. Sperling .

\section{References.}

1.P. J. Flory, Principles of Polymer Chemistry, Cornell University Press, Ithaca, (1953).

2. P. G. de Gennes, Scaling concept in polymer physics, Cornell University Press, Ithaca, (1979).

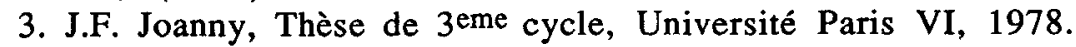

4. P. Pincus, J. Chem. Phys., 75, (1981), 1996

5. K. Binder, J. Chem. Phys., 79, (1983), 6387.

6. M. Takenaka, T. Izumitani, T. Hasimoto, J. Chem. Phys.92, (1990), 4566.

7. F.S. Bates, P. Wiltzius, J. Chem. Phys., 91, (1989), 3258. 
8. L.H. Sperling, Interpenetrating Polymer networks and related materials, Plenum Press, New York, (1981).

9. H.L. Frisch, D. Klempner, K.C. Frisch, J. Pol. Sci. A2, (1969), 7, 425.

10. P.G. de Gennes, J. Physique Lett., 40, 69, (1979).

11. R.M. Briber, B. J. Bauer, Macromolecules, 21, (1988) 3296.

12. A. Bettachy, A. Derouiche, M. Benhamou, M. Daoud, J. PhysiqueI, 1, (1991), 153.

13. M. Doi, S. F. Edwards, The theory of polymer dynamics, Oxford Science publications , (1986).

14. J. W. Cahn, Trans. Met. Soc. A.I.M.E. 242, 166, (1968).

J. E. Hilliard, in Phase transformations, H. L. Anderson ed., American Society for Metals, Metals Park, (1970).

15. K. Binder, D. W. Heermann, in Scaling phenomena in disordered solids, R. Pynn and A. Skjeltorp Eds., Plenum Press, p207, (1985).

16. P. G. de Gennes, J. Chem. Phys. 72, (1980), 4756.

17. P. G. de Gennes, J. de Physique, 42, (1981), 735. 